\title{
Mixed haematogenous endophthalmitis caused by Candida albicans and CDC fermentative corynebacterium group A-4
}

\author{
Caroline Barker, Jane Leitch, N P Brenwald, M Farrington
}

\begin{abstract}
We report a case of mixed haematogenous endophthalmitis in which Candida albicans and Centres for Disease Control (CDC) corynebacterium group A-4 were isolated together from an aspirate of vitreous humour.
\end{abstract}

\section{Case report}

A 77-year-old retired farmer was admitted to hospital in May 1987 as an emergency with a sixhour history of nausea and abdominal pain. His past medical history included a shotgun injury to his left eye resulting in enucleation at age 5, penicillin allergy, and a prostatectomy. A chest $x$-ray showed gas under the right hemidiaphragm. A perforated duodenal ulcer was oversewn at laparotomy. A Polya gastrectomy was performed six days later for persistent melaena. Postoperatively the patient became dehydrated, with a persistently high blood glucose, which responded to an insulin sliding scale and transfusion via a right jugular central venous line. The patient was transferred to the Intensive Therapy Unit, where he received total parenteral nutrition via the central line, which was in situ for 10 days. After removal the tip of the line grew $C$ albicans. At this time his peripheral white blood cell count was $29 \cdot 5 \times 10^{9} / 1$. Abdominal drain fluid grew $C$ albicans and a diphtheroid, which was not further identified, and repeated blood cultures were sterile. During the first three postoperative weeks he was intermittently febrile, and he received various combinations of gentamicin, metronidazole, cefotaxime, erythromycin, co-trimoxazole, and trimethoprim.

On the 19th postoperative day the patient complained of blurred vision in the right eye. On referral to the Ophthalmology Department his vision was $6 / 60+1$, and he was noted to have a marked anterior uveitis and an isolated area of focal chorioretinitis associated with vitreous activity. He was treated initially with topical steroids, which produced a subjective improvement. However, this was not maintained, and the posterior uveitis progressed. A lesion along the supratemporal vessels developed that was associated with beaded intravitreal abscesses. Serological tests for toxoplasma, syphilis (VDRL, TPHA), cytomegalovirus, and herpes simplex virus were negative. A presumptive diagnosis of candida endophthalmitis was made, and a vitrectomy was performed.

A Gram-stained smear of the vitreous washings showed occasional yeasts and large numbers of intracellular pleomorphic Gram- positive bacilli. In view of his deteriorating renal function the patient was treated with oral 5flucytosine $2 \mathrm{~g}$ twice daily (with regular serum assay) and ketoconazole $400 \mathrm{mg}$ once daily, with topical cefotaxime and gentamicin. A serological test for $C$ albicans performed at the Mycology Reference Laboratory, Colindale, London, was positive (precipitin titre 1:4, agglutination titre $<1: 4$ ), and the washings grew scanty yeast colonies and a heavy growth of a Gram-positive bacillus after 48 hours' incubation.

Ten days later an exudative retinal detachment began temporally and extended to become total. A vitreous washout was performed, but specimens were not sent for culture. The retinal detachment persisted, and the vision was perception of light. Over the following month a dense cataract developed, but the retinal detachment flattened spontaneously. Topical antibiotics were stopped after three weeks, and the patient was discharged nine weeks after admission on oral antifungal therapy which was stopped after a total course of four weeks. Lensectomy, vitrectomy, and division of membranes were performed when his general health had improved, resulting in improvement of vision to $6 / 36+1$. In December 1987 an inferior tractional retinal detachment was noted and repaired with injection of silicone oil.

Over the next six months vision improved to 6/ 18. The patient has had two episodes of urinary frequency with associated pyuria occurring four months and seven months after his initial admission. Urine specimens on both occasions grew $C$ albicans ( $>10^{5}$ colony forming units $/ \mathrm{ml}$ ) and symptoms resolved after short courses of oral 5-flucytosine. He has had no further ocular or urinary symptoms and is managing well with low visual aids.

\section{MICROBIOLOGY}

The yeast was identified as Candida albicans by the Mycology Reference Laboratory, Central Public Health Laboratory, Colindale, London, and found to be sensitive to 5-flucytosine, amphotericin B, ketoconazole, and miconazole. The bacterium was a strictly aerobic coccobacillus that formed yellow pigmented colonies after 48 hours' incubation on equine blood agar at $37^{\circ} \mathrm{C}$. It was non-motile at $37^{\circ} \mathrm{C}$ and room temperature, catalase and Voges-Proskauer positive, and DNAase, urease, and nitrate reductase negative. It produced acid from glucose, fructose, mannose, maltose, and mannitol but not from lactose, trehalose, xylose, sucrose, melibiose, or raffinose, and it was sensitive to penicillin and vancomycin by disc 
diffusion. It was identified by Dr Weaver, Centres for Disease Control, Atlanta, Georgia, USA as belonging to $\mathrm{CDC}$ fermentative corynebacterium group A-4.

\section{Discussion}

To our knowledge this is the first reported case of metastatic endophthalmitis caused by CDC fermentative corynebacterium group A-4, and the first case of mixed haematogenous endophthalmitis involving $C$ albicans. The majority of cases of infectious endophthalmitis follow direct inoculation by trauma or surgery. A great variety of organisms have been isolated (including $C$ albicans ${ }^{1}$ and CDC fermentative coryneform group $\mathrm{A}-4^{2}$ ), and mixed cultures are not uncommon. Haematogenous endophthalmitis, however, is much less frequent. Because of their underlying associated medical conditions these patients may present to any hospital specialty, and this may lead to delayed diagnosis and treatment. The commonest isolate is $C$ albicans, which often follows prolonged antibiotic therapy, intravenous therapy, ${ }^{3}$ or intravenous drug abuse. ${ }^{4}$ Other organisms believed to have spread to the vitreous via the blood include staphylococci, streptococci, Neisseria meningitidis, Gram-negative rods, ${ }^{5}$ mycobacteria, ${ }^{6}$ and Aspergillus fumigatus. ${ }^{7}$ A single case of mixed haematogenous endophthalmitis has been reported in which a Lancefield group $G$ $\beta$-haemolytic streptococcus and two strains of coagulase-negative staphylococci were isolated from the vitreous of a patient with streptococcal cellulitis of the leg. ${ }^{8}$

Non-diphtheria corynebacteria are components of the normal flora that pose the clinical diagnostic laboratory considerable problems of identification, and few defined clinical syndromes have yet been associated with individual species. Therefore many isolates are dismissed as contaminants. Fermentative corynebacterium CDC group A-4 has been associated with infections at many body sites including blood, cerebrospinal fluid, urine, sputum, and pleural fluid, ${ }^{10}$ but it shares with the closely related fermentative corynebacterium CDC group 1 an apparent predilection for causing endophthalmitis. Further studies on the taxonomy and clinical significance of fermentative corynebacteria are needed to define their pathogenic abilities more accurately. In our case the significance of the bacterial isolate is confirmed by its intracellular location in vitreal polymorphs and isolation in heavy growth, but its contribution to the local septic process is impossible to assess. Whereas vitrectomy is the recognised treatment for candida endophthalmitis, the additional value of systemic or local antifungal agents is disputed, but the balance of opinion favours the use of systemic 5-flucytosine and ketoconazole. ${ }^{3}$ Although suffering a mixed fungal and coryneform infection, our patient responded well to vitrectomy, oral antifungal agents, and topical antibiotics.

Candida endophthalmitis is a largely avoidable iatrogenic complication. Candidaemia is rarely suspected or detected at the time it occurs, but repeated questioning about visual disturbance in patients treated with antibiotics and indwelling intravenous catheters who become febrile may alert the clinician to the presence of endophthalmitis at an early stage in its development.

We thank Mr D W Flanagan for permission to report this case, and Professor D W R MacKenzie (Mycology Reference Laboratory, Central Public Health Laboratory, Colindale, London) and Dr R E Weaver (Director, Special Bacteriology Section, Centres for Disease Control, Atlanta, Georgia, USA) for confirming the identity of the organisms.

1 Forster RK. Endophthalmitis, diagnostic cultures and visual results. Arch Ophthalmol 1974; 92: 387-92.

2 Weber DJ, Haffman KL, Thoft RA, Baker AS. Endophthalmitis following intraocular lens implantation: report of 30 cases and review of the literature. Rev Infect Dis 1986;8: 129.

3 Graham E, Chignell AG, Eykyn S. Candida endophthalmitis: a complication of prolonged intravenous therapy and antibiotic treatment. F Infect 1986; 13: 167-73.

4 Salmon JF, Partridge BM, Spalton DJ. Candida endophthalmitis in a heroin addict. Br $\mathcal{F}$ Ophthalmol 1983; 67: 306-9.

5 Farber BP, Weinbaum DL, Dummor JS. Metastatic bacterial endophthalmitis. Arch Intern Med 1985; 145: 62-4.

6 Lester H, Erdey RA, Fastenberg DM, Schwanz PL, Rosenhaus JB. Bacillus Calmette-Guérin endophthalmitis. Retina 1988; 8: 182-4.

7 Weiss JN, Hutchins RK, Balogh K. Simultaneous aspergillus endophthalmitis and cytomegalovirus retinitis after kidney transplantation. Retina 1988; 8: 193-8.

8 Puliafito CA, Baker AS, Haaf J, Foster CS. Infectious endophthalmitis: review of 36 cases. Ophthalmology 1982; 89: $921-9$.

9 Na'was TE, Hollis DG, Moss CW, Weaver RE. Comparison of biochemical, morphologic and chemical characteristics of Centres for Disease Control fermentative coryneform groups 1,2 and A-4. F Clin Microbiol 1987 25: 1354-8.

10 Hollis DG, Weaver RE. Gram-positive organisms: a guide to identification. Atlanta, Georgia: Centres for Disease Control, 